\title{
On the Skewness of the Sea Slope Probability Distribution
}

\author{
Bertrand Chapron \\ IFREMER, Département Océanographie Spatiale, Plouzané, France \\ D. Vandemark \\ NASA/Goddard Space Flight Center, Wallops Island, Virginia USA
}

T. Elfouhaily

Johns Hopkins University, Applied Physics Laboratory, Laurel, Mariland USA

\begin{abstract}
We revisit skewness observations for the sea slope probability density function as deduced using optical glitter measurements collected in the 1950s. This slope skewness was addressed by Longuet-Higgins who concluded that localization of short steep wavelets along the phase of underlying longer waves was the most likely physical cause for the phenomena. The present work suggests that the actual wave form under near-breaking conditions, along with the varying population and length scales for these breaking events, should also contribute to the skewness. This latter component is a larger factor for moderate to high winds while the former should dominate at light winds. It is suggested that Cox and Munk's skewness estimate can be related to the modeling and remote sensing of wave breaking probability and perhaps to quantifying the critical role that wave dissipation plays within air-sea gas transfer.
\end{abstract}

\section{INTRODUCTION}

An area of unfilled promise in ocean remote sensing is the development of a consistent inversion of sea surface short wave characteristics via the ever-increasing complement of microwave and optical techniques. Such an interest stems largely from the potential of these measurements to provide information on geometrical and kinematic properties of the sea surface that can be re-

Gas Transfer at Water Surfaces

Geophysical Monograph 127

Copyright 2002 by the American Geophysical Union lated to physical ocean surface processes, such as the rate of gas exchange across the sea surface.

For instance, there is increasing experimental evidence that breaking waves, which impact exchanges of momentum, heat and gas, do contribute strongly to certain remote sensing measurements. From a statistical viewpoint, wave breaking events occur infrequently. These events are termed sporadic or intermittent, and are difficult to quantify. Geometrically, the wave profile under breaking conditions is typically characterized with asymmetry and steepness approaching a large critical value. The tools necessary for precise field determinations remain in development, nonetheless individual breaking events at a multitude of length scales are 


\section{0) SEA SLOPE SKEWNESS}

likely contributors to distinct signatures in optical and microwave remote sensors:

This note is primarily focused on issues pertaining to the inference of the ocean suface slope statistics using tiechniques that rely upon the quasi-specular reftection mechanism. The initial assumption often invoked to interpret experimental data in such studies is Gaussianity in the surface slope probability distribution function (PDF). However, it is quite clear from the seminal Cox and Munk sun glitter measurements and their reported parameters [Cox and Munk, 1956] that the slope PDF exhibits substantial third and fourth order corrections with respect to a Normal distribution [Cox and Munk, 1956; Longuet-Higgins, 1982]. One immediate impact of non-Gaussian wave slope statistics is its place in modifying the predicted occurrence of exceedingly steep and/or asymmetric slope values. A better identification of these statistical moment signatures within remote sensing measurements [e.g. Chapron et al., 2000; Liu et, al., 1997] should help to clarify the relation between these parameters and air-sea gas exchange, as well as the development of possible strategies to parameterize these exchanges in terms of remote sea surface geometrical inferences.

Recently, even-order moments of the slope PDF were addressed [Chapron et al., 2000] to show that observed distribution kurtosis is wholly consistent with a two scale model for the ocean surface. This study indicated that the commonly invoked 'long-wave short-wave interaction' concept readily leads to a non-Gaussian, peaked distribution. The present, study concentrates on the skewness of the sea slope distribution and revisits the work of Longuet-Higgins (1982) which in turn starts from Cox and Munk's (1956) observations. The primary objective is to take another look at the physical explanation for the observed skewness.

\section{OPTICALLY-DEDUCED SEA SURFACE SLOPE DISTRIBUTIONS}

The intensity of sun glitter versus the viewing angle was used by Cox and Munk for their slope PDF estimations. The technical limitations of that study have been well documented. As recently re-evaluated [Chapron et; al., 2000], the authors could not estimate the time moments of the distributions due to lack of normalization. They were left to approximate the distribution statistics using observations with an angular limit that left uncertainty in those estimates [Wentz, 1976]. That analysis utilized a polynomial fit to capture the non-Gaussianity and directionality they observed in the datia. The re- gression was performed on the natural logarithm of their intensity observations versus view angle, written as

$$
\begin{aligned}
\log (p)= & a_{o}-a_{o}^{\prime} s^{2}+a_{o}^{\prime \prime} s^{4} \\
& +s\left(a_{1}-a_{1}^{\prime} s^{2}\right) \cos \alpha^{\prime} \\
& +s^{2}\left(a_{2}+a_{2}^{\prime} s^{2}\right) \cos 2 \alpha^{\prime} \\
& +a_{3} s^{3} \cos 3 \alpha^{\prime}+a_{4} s^{4} \cos 4 \alpha^{\prime} \\
& \text { and } a_{o}^{\prime} s^{2} \leq 4
\end{aligned}
$$

with $s$ the slope value, $\alpha^{\prime}$ the azimuthal angle according to principal axis (the wind direction), and $a_{n}^{m}$ the fit coefficients. These coefficients were then related to the expansion coefficients pertaining to the cumulants of the slope distribution under a Gram-Charlier approximation. Note that; $a_{0}$ in Eq. 1 is an arbitrary offset value due to the inability to fully resolve the steepest angles.

As developed, two key parameters emerge with respect to non-Gaussianity. These are the ratio $a_{o}^{\prime \prime} / a_{o}^{\prime 2}$ and the odd-order coefficient; $a_{1}$. A non-zero value for the former indicates that the slope PDF exhibits a substantial departure from a parabolic fit approximation corresponding to a pure Gaussian distribution. Cox and Munk did find this term to be non-negligible and indicative of a distribution peakedness. We refer the reader to Chapron et al. [2000] for a recent re-interpretation of these results and their physical relevance. For the present paper we wish to recall that the peakedness can be directly attributed to the modulation of shorti-scale wavelets by a random underlying longer-scale processes such as the long wave field, its varying steepness, wind gustiness etc... Moreover, that paper notes the simple, yet perhaps subtle, issues involved when converting from Cox and Munk's observed polynomial coefficients to distribution moments for the case where exact values for variances and higher-order moments are of interest.

A non-zero value for the odd-order $a_{1}$ coefficient indicates an angular shift of the most-likely specular: point. The study of Longuet-Higgins (1982) provides a robust examination of this optically-derived evidence for skewness of the surface slopes based upon the case study data of Cox and Munk (1956). As observed, the mode of the slope distribution did not occur at zero slope for the case of wind-driven slope data collected looking along the wind direction. As reported, the most-likely slope value is of the order $3-4^{\circ}$ towards the downwind. This apparent 'skewness', or shift, angle $(\Delta)$ was not observed looking in the crosswind direction or for the case where oil was used to damp out cm-scale waves.

Longuet-Higgins computed a first-order development, using the Gram-Charlier approximation that permits a link between observed $\Delta$ values and the coefficient of 


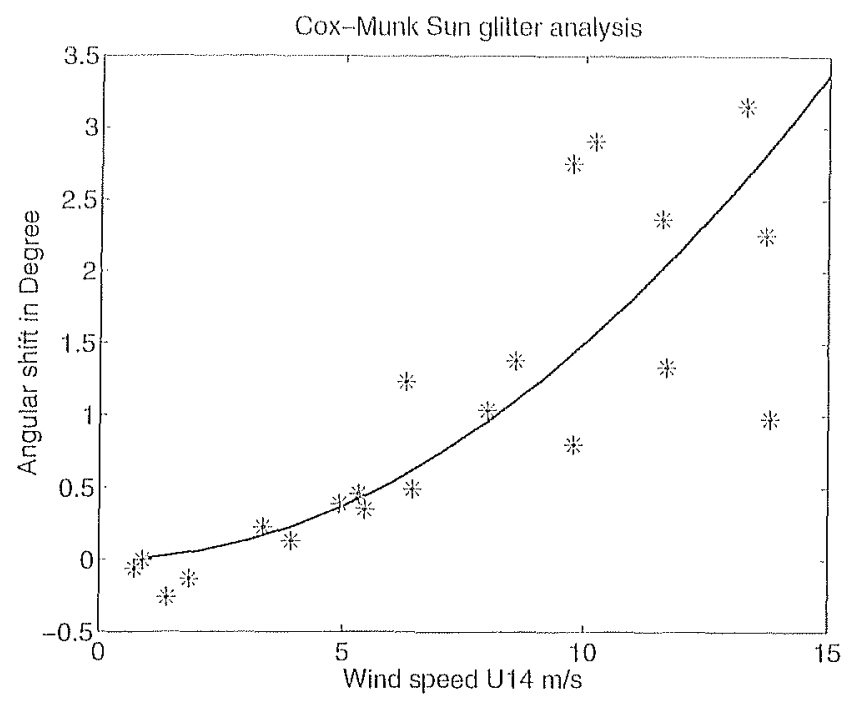

Figure 1. Mosti-likely specular points as deduced from Cox and Munk fit parameter.

skewness $\left(\lambda_{3}\right)$. We would propose a slightly modified, but nearly equivalent, approach where according to Eq. 1, the maximum intensity would approximately occur at an angle given by

$$
\Delta \simeq \frac{a_{1}}{2\left(a_{o}^{\prime}-a_{2}\right)}
$$

We suggest that since the total variance is not directly estimated, an interpretation of this shift angle in terms of the skewness statistical parameter is not straightforward. A more precise rendering normalizes $a_{1}$ by the along-wind incomplete variance, $1 /\left(a_{o}^{\prime}-a_{2}\right)$. This will result in somewhat smaller values compared to the initial study conducted by Longuet-Higgins (1982). Regardless, the non-zero shift angle and its dependence on the mean-square-slope (i.e. wind speed for the Cox and Munk case) are still clearly in evidence as shown in Figure 1.

\section{MODELING NON-GAUSSIAN SEA SURFACE SLOPES}

Several questions are posed within Longuet-Higgins (1982). The majority of this effort deals with modeling related to this question: What is the physical explanation for the observed skewness? The study examines several possibilities to explain the observed angular shift. The first approach is to consider the skewness of the individual waves where one assumes the existence of a shifted, bound second hamonic that distorts the lineas: form. Such a geometry should only occur in a transient statie, just before breaking. The author concludes that this effect may be relevant to longer gravily waves but its direct application to the range of high-frequency waves that dominate the slope PDF is somewhat unclear. A second possibility of skewness in waves when considering viscous dumping is dismissed due to the very weak computed impact. The study's search for physical explanation ends with the assumption of a deterministic two scale model in which capillary-gravity waves, contributing the most to the slope variance, are modulated in phase and amplitude by a fast-propagating but slowly varying longer wave. The author concludes that this last phenomenon leads to plausible agreement with the data in tems of magnitude and sign for $\Delta$. Fxplicit in this choice is the realization that slope skewness is directly associated with long wave steepness and propagation direction and thus there is no necessary fundamental relation between the wind stress and distribution skewness. Moreover, the sign can change if the angle between wind waves and swell were to approach $\pi$.

We postulate that this deterministic and hydrodynamic model for the skew effect; should be rejoined with that study's first possibility - that the wave's themselves can be skewed under near-breaking conditions. Moreover, the source of short-wave modulation can be of both hydrodynamical and aerodynamical origins. Theoretically and experimentally, it is known that modulational and direct wind input effects produce shortening and steepening of the shorter waves. The two main physical explanations mentioned above can thus both contribute to the resulting non-Gaussian slope statistics.

Undoubtedly, near-breaking wavelets can locally dominate the slope variance (e.g. sea spikes). Consequently, the non-Gaussian statistics can be hypothesized to result from the modulated occurrence of steep asymmetric short waves along longer wave profiles. This modulated occurrence contributes to an apparent modulation of the slope variance (between non-breaking and breaking short wave packets). If such intermittencies and groupiness are sufficiently sampled, a compound model may be considered to describe the surface slope statistics [Chapron et al., 2000]. Following such a model, all sources of non-stationarities and modulations, including wave-wave interactions, wind input modulation, local breaking, leads one to consider the slope variance as a random variable. Mathematically, such a compound model leads at the lowest even orders to an expression congruent with the empirical shape analysis proposed by Cox and Munk, i.e. Eq. 1. Under such a development, the departure from Gaussianity is explicitly 
associated with the rariance of the mean square slope fluctuations, and will thus control the value of the ratio $a_{o}^{\prime \prime} / a_{o}^{\prime 2}$.

To augment the hydrodynamical approach of LonguetFiggins, one can then follow the phenomenological approach introduced by Phillips [Phillips, 1985] to consider the skewness contribution associated with nearbreaking waves. The evaluation can be made by considering the average total length of breaking fronts at a given scale, $\Lambda(\mathrm{k})$. The breaking fronts are conceptually associated with isolated irregular line segments with discontinuous asymmetric slope changes. To simplify the description, the sharp crested near-breaking waves are further assumed to have self-similar asymmetric shapes (bound phase-shifted higher harmonics). Larger breaking waves are then simply magnified copies of smaller breakers.

The total average surface covered by sharp crested waves at a given scale per unit surface will be propotional to

$$
d A(k) \propto \frac{\Lambda(k)}{k} d k
$$

leading to an expected total slope asymmetry proportional to

$$
\Delta \propto \Delta_{i} \int \frac{\Lambda(k)}{k} d k
$$

where $\Delta_{i}$ is the self-similar asymmetry of an individual self-similar transient. As suggested by Phillips, the integration covers the gravity range. This range is limited at high wavenumber to scale whose phase speed is not small compared with the friction velocity $u_{*}$, i.e.

$$
h_{\max } \leq \min \left\{(g / \gamma)^{1 / 2}, g / u_{*}^{2}\right\}
$$

with $\gamma$ the surface tension. Indeed, in the range of small gravity waves $(\leq 15-20 \mathrm{~cm}$ and $\geq 3-5 \mathrm{~cm}$ ), the dissipation will be dominated by the generation of parasitic capillaries [Kudryavtsev et al., 1999].

According to Phillips, $\Lambda(k)$ should vary as $u_{\star}^{3} k^{1 / 2}$, leading to $\Delta \propto u_{*}^{2}$ which is consistent with the Cox and Munk's very short fetch glitter observations (see Figure 1). Under this phenomenological approach, it can also be postulated that over the total average surface covered by active wave-breaking events, the surface slope variance along the wind direction is necessarily limited to a critical constant maximum valıe. The along-wind slope variance should thus also vary as $u_{\star}^{2}$.

However, under open sea conditions, $\Lambda(k)$ and the total fraction of the surface covered by sporadic breaking events will also depend upon be non-wind environmental conditions (e.g. swell amplitude and directionality, current, atmospheric stability, slicks). These effects will likely limit a direct identification between the shift angle and the wind stress.

The combination of the two-scale hydrodynamic model with the multi-scale breaking phenomena provides two skewness-generating processes that are in play over most. of the ocean most of the time. Their relative contribution or precedence will vary and they are also, in some sense, inseparable. However, one anticipates that the direct long wave modulation effect will be most evident at lighter winds where field observations of the modulation transfer function show a trend running from a. maximum at lightest winds, decreasing with $u_{x}$. Wave breaking and thus actual wave geometry skew will generally increase in proportion to the population of breaking events, and corresponding increase of the range of length scales involved in brealking. We reiterate that some combination of hydrodynamic and aerodynamic modulation along the longer waves is likely fundamental to the breaking environment. Nonetheless, as described above one can expect that, to first-order, the average fraction of the surface covered by active wave breaking events will scale with $u_{*}^{n}$ in a manner that may likely follow field-observed trends for wave breaking at micro and macro-scales.

\section{CONCLUSION}

With an overall objective to refine links between ocean surface processes and remote sensing measurements, it is necessary to have a precise sea surface slope statistical description. In many studies, Gaussian statistics are a leading assumption. This first-order approximation is numerically attractive and requires only the variance for a complete model. However, optical scattering measurements are shown to consistently indicate the need to report at least two parameters in an even-order statistical analysis, in addition to a systematic angulax clisplacement of the most intense glitter reflection. A physical interpretation for observing infrequent steep asymmetric slopes can be given via considering the modulated occurrence of near breaking waves.

Numerous studies (e.g. Longuet-Higgins, 1982; Walsh et al. 1998; Shaw and Churnside, 1997; Liu et al., 1997) suggest that high frequency radar and/or optical techniques applied at near-nadir incidence angles should lead to further clarifications of the physics and correlatives involved in determining the slope distribution. But from the presnt ananlysis, it is stressed that the data-fitting procedures must include up to fourth order corrections. This is necessary to properly determine, as a minimum, the slope variance. 
We anticipate that one end goal for such data should be a better detemination of wave breaking statistics, and improved quantification of the role of sharply crested waves in gas transfer across the sea surface.

\section{REFERENCES}

Chapron B., V. Kerbaol, D. Vandemark and T. Elfouhaily, Importance of peakedness in sea surface slope measurements and applications, J. Geophys. Res, 105, 1719517202,2000

Cox, C. and W. Munk, Slopes of the sea surface deduced from photographs of sun glitter, Bull. Scripps Inst. Occan., 6, 9, 401-488, 1956.

Kudryavtsev, V.N., V.K. Makin and B. Chapron, Coupled sea surface-atmosphere model 2. Spectrum of short wind waves, J. Geophys. Res., 104, 7625-7639, 1999.

Liu Y., X.-H. Yan, W.T. Liu, and P.A. Hwang, The probability density function of ocean surface slopes an its effect on radar backscatter, J. Phys. Oceanogr., 25, 782-797, 1997.

Longuet-Higgins, M.S., On the skewness of sea surface slopes, J. Phys. Occan., 12, 1283-1291, 1982.
Phillips, O.M., Spectral and statistical properties of the equilibrium range in wind-generated gravity waves, $J$. Fluid Mech, 156, 505-531, 1985.

Shaw, J. and J. Churnside, Scanning laser glint measurements of sea-surface slope statistics, Appl. Optics, 36 , $4202-4213,1997$.

Walsh, E. D., D. Vandemark, C. Friehe, S. Burns, and D. Khelif, Measuring sea surface mean square slope with a 36-GHz scanning radar altimeter, J. Geophys. Res., 103, 12613-12628, 1998.

Wentz, F., Cox and Munk's sea surface slope variance, $J$. Geophys. Res., 81, 1607-1608, 1976.

B.Chapron, IFREMER, Département: Océanographie Spatiale, 29280 Plouzané, France (e-mail: bchapron@ifremer.fr)

D. Vandemark, NASA Goddard Space Flight Center, Laboratory for Hydrospheric Processes, Wallops Island, 23337, VA, USA (e-mail: vandemark@gsfc.nasa.gov)

T. Elfouhaily, The Johns Hopkins University, Applied Physics Laboratory, 11100 Johns Hpkins road, Lanrel, MD 20723-6099, USA (e-mail: Elfouhaily@jhuapl.edu) 